\title{
Obesity inflames memory circuits
}

Obesity may have negative effects on the brain regions involved in learning and memory. The underlying mechanisms remain to be fully elucidated, but indirect evidence suggests that pro-inflammatory cytokines contribute to these effects. Now, Stranahan and colleagues show that adipose-linked interleukin- $1 \beta$ (IL-1 $\beta$ ) mediates memory deficits in a mouse model of obesity.

The authors studied leptin receptor-deficient mice ( $d b / d b$ mice), which develop an obese phenotype because of leptin insensitivity. They found that sedentary $d b / d b$ mice performed worse in spatial and object memory tasks than sedentary wild-type animals and displayed deficits in hippocampal long-term potentiation, as revealed from recordings in the dentate gyrus. Immunofluorescence analysis revealed that sedentary $d b / d b$ mice also had fewer hippocampal dendritic spines. Strikingly, these deficits were attenuated in $d b / d b$ mice that underwent daily treadmill training for 3 months, indicating that obesity - rather than leptin insensitivity - adversely affects hippocampal function.

$d b / d b$ mice exhibit systemic inflammation, and the authors found that treadmill training lowered the expression of major histocompatibility complex II (a marker of activated microglia and macrophages) in the hippocampus and in adipose tissue of these animals. Such training was also associated with lower levels of circulating, fat pad and hippocampal IL-1 $\beta$, suggesting that an inflammatory mechanism may mediate the effects of obesity on learning and memory.

Interestingly, sedentary lipectomized $d b / d b$ mice performed markedly better on memory tasks and exhibited higher levels of dendritic synaptic markers than sedentary $d b / d b$ animals that underwent sham surgery. By contrast, wild-type mice that received a fat pad transplant from sedentary $d b / d b$ mice exhibited memory deficits and a loss of synaptic markers. Furthermore, lipectomy inhibited, and fat transplant promoted, the production of inflammatory markers, including IL- $1 \beta$, as revealed ex vivo in hippocampal homogenates and in vitro in isolated monocytes stimulated with lipopolysaccharide. Together, these findings indicate that adipose-associated inflammation impairs hippocampal function.

Levels of IL- $1 \beta$ correlated well with body fat percentage and the loss of hippocampal function in the treadmill and fat transplant studies, so the authors examined whether this cytokine could link obesity to hippocampal dysfunction. They blocked IL- $1 \beta$ function in sedentary $d b / d b$ and wildtype mice by hippocampal infusion of IL-1ra, an IL-1 receptor antagonist. IL-1 ra infusion had no effect on memory or the expression of synaptic markers in wild-type mice, but in $d b / d b$ mice, it abolished the memory and long-term potentiation deficits and restored dendritic spinal densities. These findings suggest that IL- $1 \beta$ may mediate the negative consequences of obesity on hippocampal function in these animals.

This study reveals a mechanism through which obesity may affect learning and memory circuits. The authors suggest that adipose tissuederived cytokines such as IL- $1 \beta$ can enter the brain to cause local hippocampal inflammation and synaptic dysfunction, which in turn may result in cognitive impairment.

Natasha Bray

ORIGINAL RESEARCH PAPER Erion, J. R. et al. Obesity elicits interleukin 1-mediated deficits in hippocampal synaptic plasticity.J. Neurosci. 34, 2618-2631 (2014) wild-type mice that received a fat pad transplant from sedentary $d b / d b$ mice exhibited memory deficits 